\title{
Faithful simple objects, orders and gradings of fusion categories
}

\author{
SONIA NATALE
}

\begin{abstract}
We establish some relations between the orders of simple objects in a fusion category and the structure of its universal grading group. We consider fusion categories that have a faithful simple object and show that their universal grading groups must be cyclic. As for the converse, we prove that a braided nilpotent fusion category with cyclic universal grading group always has a faithful simple object. We study the universal grading of fusion categories with generalized Tambara-Yamagami fusion rules. As an application, we classify modular categories in this class and describe the modularizations of braided Tambara-Yamagami fusion categories.
\end{abstract}

18D10, 16T05

\section{Introduction}

Group gradings on fusion categories and more precisely, group extensions of a fusion category, are key ingredients in several classification results. In particular, they underlie the notions of nilpotency and solvability of a fusion category, developed in Gelaki and Nikshych [14], and Etingof, Nikshych and Ostrik [12]. Group extensions of fusion categories have been recently classified in Etingof, Nikshych and Ostrik [11].

We shall work over an algebraically closed base field $k$ of characteristic zero. Let $\mathcal{C}$ be a fusion category over $k$. There is a canonical faithful grading $\mathcal{C}=\bigoplus_{g \in U(\mathcal{C})} \mathcal{C}_{g}$, called the universal grading of $\mathcal{C}$, with trivial homogeneous component $\mathcal{C}_{e}=\mathcal{C}_{\text {ad }}$, where $\mathcal{C}_{\text {ad }}$ is the adjoint subcategory of $\mathcal{C}$, that is, the fusion subcategory generated by $X \otimes X^{*}$, where $X$ runs through the simple objects of $\mathcal{C}$ (Etingof, Nikshych and Ostrik [10]). The group $U(\mathcal{C})$ is called the universal grading group of $\mathcal{C}$ [14].

Let $X$ be an object of $\mathcal{C}$. Then $X$ is called faithful if the fusion subcategory $\mathcal{C}[X]$ generated by $X$ is all of $\mathcal{C}$, so that $X$ is faithful if and only if every simple object of $\mathcal{C}$ appears with positive multiplicity in some tensor power of $X$.

Let $G$ be a finite group. An example of a fusion category over $k$ is given by the category $\operatorname{rep} G$ of finite dimensional $k$-linear representations of $G$. In this case, the 
universal grading group is isomorphic to the dual group $\widehat{Z(G)}$ of the center $Z(G)$ of $G$ and the adjoint subcategory coincides with the category $\operatorname{rep} G / Z(G)$. See [14].

Suppose $V$ is a finite-dimensional representation of $G$, and let $\chi$ be the character of $V$. Then it follows from the Burnside-Brauer Theorem (see Isaacs [16, Theorem 4.3]) that $V$ is a faithful object of $\operatorname{rep} G$ if and only if $\chi$ is a faithful character of $G$, that is, if and only if $\operatorname{ker} \chi=1$.

Let $X$ be a nonzero object of a fusion category $\mathcal{C}$. The order of $X$ is the smallest natural number $n$ such that the $n^{\text {th }}$ tensor power $X^{\otimes n}$ contains the trivial object $\mathbb{1}$ of $\mathcal{C}$. The order of every nonzero object in $\mathcal{C}$ is finite; indeed, it is not bigger than the rank of $\mathcal{C}$. This invariant is introduced and studied in Kashina, Sommerhäuser and Zhu [17] for the category of representations of a semisimple Hopf algebra.

The orders of simple objects play a rôle in recent classification results Rowell, Stong and Wang [25], and Hong and Rowell [15]. As pointed out in [15], the classification of modular categories of a given rank divides naturally into those for which every simple object is self-dual, that is, of order at most 2 , and those for which at least one simple object is not self-dual, that is, of order bigger than 2 . See, in particular, Theorem 2.2 of [loc. cit.].

In this paper we establish some relations between the structure of the group $U(\mathcal{C})$ and the orders of the simple objects of $\mathcal{C}$. We show that if $\mathcal{C}$ is generated by simple objects $X_{1}, \ldots, X_{m}$, then the group $U(\mathcal{C})$ is generated by elements $g_{1}, \ldots, g_{m}$, such that $X_{i} \in \mathcal{C}_{g_{i}}$, and the order of $g_{i}$ divides the order of $X_{i}$, for all $i=1, \ldots, m$. Hence, if $\mathcal{C}$ has a faithful simple object $X$, then the universal grading group of $\mathcal{C}$ is cyclic and its order divides the order of $X$.

We also establish the converse in the case where $\mathcal{C}$ is braided and nilpotent. That is, if such a fusion category has a cyclic universal grading group, then it has a faithful simple object. These results are contained in Section 4; see Theorems 4.1 and 4.7. They extend classical results for finite groups. The proof of Theorem 4.7 relies on the one hand on Theorem 2.1, which generalizes, in the braided case, the fact that a nontrivial normal subgroup of a finite nilpotent group intersects the center nontrivially, and on the other hand, on the fact that every braided nilpotent fusion category is equivalent to a tensor product of braided fusion categories whose Frobenius-Perron dimensions are powers of distinct primes (Drinfeld, Gelaki, Nikshych and Ostrik [8, Theorem 1.1]).

Let us summarize some consequences of these facts. See Corollaries 4.3, 4.4, 4.9 and Proposition 4.8. Firstly, for any fusion category $\mathcal{C}$, the exponent of $U(\mathcal{C})$ divides the least common multiple of the orders of simple objects of $\mathcal{C}$. Thus, if every simple object is self-dual, then $U(\mathcal{C})$ is an elementary Abelian 2-group. 
Also, if $\mathcal{C}$ is nilpotent and has a simple object of order $p$, where $p$ is a prime number, then the Frobenius-Perron dimension of $\mathcal{C}$, which is always an integer, is divisible by $p$. Moreover, if the order of every simple object of $\mathcal{C}$ is a power of $p$, then the Frobenius-Perron dimension of $\mathcal{C}$ is a power of $p$.

Assume in addition that $\mathcal{C}$ is braided. If $X_{1}, \ldots, X_{n}$ are simple objects that generate $\mathcal{C}$ as fusion category, we get that the exponent of $U(\mathcal{C})$ divides the least common multiple of the orders of $X_{1}, \ldots, X_{n}$. In particular, $U(\mathcal{C})$ is an elementary Abelian 2-group if $\mathcal{C}$ is generated by self-dual simple objects.

These results can be formulated in terms of the group of invertible objects of $\mathcal{C}$ when $\mathcal{C}$ is a modular category, since in this case this group is dual to the group $U(\mathcal{C})$ [14].

In the last two sections of the paper we discuss some applications of the relations between the orders of simple objects and the orders of the elements of the universal grading group to study a class of fusion categories. We consider fusion categories $\mathcal{C}$ with generalized Tambara-Yamagami fusion rules in the sense that $\mathcal{C}$ is not pointed and the tensor product of two simple objects of $\mathcal{C}$ is a sum of invertible objects. Fusion categories with these kind of fusion rules are classified, up to equivalence of tensor categories, in Liptrap [18].

In Section 5 we discuss the universal grading of this kind of fusion categories. We then apply the results of this section to establish a classification result for modular categories in this class. Specifically, we show that if $\mathcal{C}$ is a modular category, then $\mathcal{C}$ has generalized Tambara-Yamagami fusion rules if and only if $\mathcal{C}$ is equivalent to $\mathcal{I} \otimes \mathcal{B}$, where $\mathcal{I}$ is an Ising category and $\mathcal{B}$ is a pointed modular category. See Theorem 5.4. This implies the classification of such modular categories in terms of group theoretical data, in view of the results on Ising and pointed modular categories Drinfeld, Gelaki, Nikshych and Ostrik [9, Section 2.11 and Appendix B], and Quinn [24].

Let $\mathcal{C}$ be a Tambara-Yamagami fusion category. Up to isomorphism, $\mathcal{C}$ has exactly one non-invertible object $X$ and a (necessarily Abelian) group of invertible objects $G$ such that $X \otimes X \simeq \bigoplus_{g \in G} g$. The classification of these categories, up to tensor equivalence, is given in Tambara and Yamagami [28]. The possible structures of a braided and spherical fusion category in $\mathcal{C}$ are classified in Siehler [26]. By [26, Theorem 1.2], $\mathcal{C}$ admits a braiding if and only if $G$ is an elementary Abelian 2-group. In particular, FPdim $\mathcal{C}=2^{m+1}$, where $m$ is the rank of $G$. Moreover, by [26, Theorem 1.2 (3)] each braiding of $\mathcal{C}$ has two choices of ribbon structures compatible with it.

Let $\mathcal{C}$ be a braided Tambara-Yamagami fusion category and let us regard $\mathcal{C}$ as a pre-

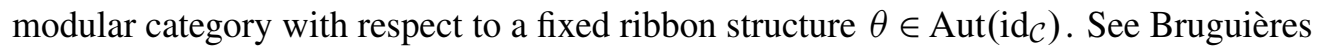
[5] and Müger [19]. 
We show that $\mathcal{C}$ is modularizable and describe its modularization $\tilde{\mathcal{C}}$. We prove that $\tilde{\mathcal{C}}$ is pointed if and only if $\mathcal{C}$ is integral. Otherwise, $\widetilde{\mathcal{C}} \simeq \mathcal{I}$, where $\mathcal{I}$ is an Ising category. This is contained in Section 6. Theorem 6.4 is the main result of this section. Its proof relies on the fact that, unless it is symmetric, $\mathcal{C}$ is a $\hat{T}$-equivariantization of $\widetilde{\mathcal{C}}$ for a certain subgroup $T$ of index at most 2 of the group $G$ of invertible objects of $\mathcal{C}$.

Recall from Siehler [27] that if $G$ is a finite group and $\kappa$ is a nonnegative integer, a near-group fusion category of type $(G, \kappa)$ is a fusion category $\mathcal{C}$ whose isomorphism classes of simple objects are represented by $G$ and a non-invertible object $X$, obeying

$$
g \otimes h \simeq g h, \quad X \otimes X \simeq \bigoplus_{g \in G} g \oplus \kappa X, \quad \forall g, h \in G .
$$

Near-group categories of type $(G, 0)$ are thus Tambara-Yamagami categories.

For the type $(G, \kappa)$ with $\kappa>0$, all possible structures of braided fusion category in $\mathcal{C}$ are classified in Thornton [29]. In this case, if $\mathcal{C}$ is not symmetric, then either $G=1$ or $G$ is isomorphic to $\mathbb{Z}_{2}$ or $\mathbb{Z}_{3}$. It follows also from the results of [29] that any braided near-group category is a premodular category and, as such, it is modularizable. Furthermore, the modularization of $\mathcal{C}$ is a pointed fusion category, unless $\mathcal{C}$ is of type $(1,1)$, also called a Yang-Lee category, in which case $\mathcal{C}$ is modular.

It turns out that the modularization of any braided near-group category is always either pointed or self-dual of rank at most 3 .

\section{Acknowledgements}

This work was partially supported by CONICET and SeCYT-UNC. The author thanks the referee for his/her useful comments and suggestions that simplified several proofs in the original manuscript.

\section{Conventions and notation}

Let $\mathcal{C}$ be a fusion category over $k$. That is, $\mathcal{C}$ is a $k$-linear semisimple rigid tensor category $\mathcal{C}$ with finitely many isomorphism classes of simple objects, finite-dimensional spaces of morphisms and such that the unit object $\mathbb{1}$ of $\mathcal{C}$ is simple. The set of isomorphism classes of simple objects in $\mathcal{C}$ will be denoted by $\operatorname{Irr}(\mathcal{C})$. By abuse of notation, we shall indicate a simple object and its isomorphism class by the same letter. In analogy with the notation used in [16, Chapter 12] in the case of finite group representations, we shall indicate by $\operatorname{cd}(\mathcal{C})$ the set of Frobenius-Perron dimensions of simple objects of $\mathcal{C}$.

If $X \in \operatorname{Irr} \mathcal{C}$ and $Y$ is an object of $\mathcal{C}$, the multiplicity of $X$ in $Y$ will be denoted by $m(X, Y)$. Thus $m(X, Y)=\operatorname{dim}_{\operatorname{Hom}_{\mathcal{C}}}(X, Y)$ and $Y \simeq \bigoplus_{X \in \operatorname{Irr}(\mathcal{C})} m(X, Y) X$. 
The group of isomorphism classes of invertible objects of $\mathcal{C}$ will be denoted by $G(\mathcal{C})$. The group $G(\mathcal{C})$ acts on the set $\operatorname{Irr}(\mathcal{C})$ by left (and right) tensor multiplication. Denote by $G[X]$ the stabilizer of $X \in \operatorname{Irr}(\mathcal{C})$ under the left action of $G(\mathcal{C})$. If $g \in G(\mathcal{C})$ and $X \in \operatorname{Irr}(\mathcal{C})$, we have $g \in G[X]$ if and only if $m\left(g, X \otimes X^{*}\right)>0$ if and only if $m\left(g, X \otimes X^{*}\right)=1$.

A fusion subcategory of $\mathcal{C}$ is a full tensor subcategory stable under direct sums and subobjects. A fusion subcategory is itself a fusion category [9, Corollary F.7]. Let $S$ be a set of objects of $\mathcal{C}$. The smallest Abelian subcategory of $\mathcal{C}$ containing $S$ and stable by direct sums and subobjects will be denoted $(S)$. We shall indicate by $\mathcal{C}[S]$ the fusion subcategory of $\mathcal{C}$ generated by $S$. The fusion subcategory generated by $\{X\}$, where $X$ is an object of $\mathcal{C}$, will be denoted $\mathcal{C}[X]$. The maximal pointed fusion subcategory of $\mathcal{C}$ is denoted $\mathcal{C}_{\mathrm{pt}}=\mathcal{C}[G(\mathcal{C})]$.

\section{Group gradings on fusion categories}

Let $\mathcal{C}$ be a fusion category and let $G$ be a finite group. A $G$-grading on $\mathcal{C}$ is a decomposition of $\mathcal{C}$ as a direct sum of full Abelian subcategories $\mathcal{C}=\bigoplus_{g \in G} \mathcal{C}_{g}$, such that $\mathcal{C}_{g}^{*}=\mathcal{C}_{g^{-1}}$ and the tensor product $\otimes: \mathcal{C} \times \mathcal{C} \rightarrow \mathcal{C}$ maps $\mathcal{C}_{g} \times \mathcal{C}_{h}$ to $\mathcal{C}_{g h}$. The neutral component $\mathcal{C}_{e}$ is thus a fusion subcategory of $\mathcal{C}$.

A $G$-grading on $\mathcal{C}$ is equivalently determined by a function $\lambda: \operatorname{Irr}(\mathcal{C}) \rightarrow G$ such that $\lambda\left(X^{*}\right)=\lambda(X)^{-1}$ and $\lambda(Z)=\lambda(X) \lambda(Y)$, for all $X, Y, Z \in \operatorname{Irr}(\mathcal{C})$ such that $m(Z, X \otimes Y)>0$.

The grading $\mathcal{C}=\bigoplus_{g \in G} \mathcal{C}_{g}$ is called faithful if $\mathcal{C}_{g} \neq 0$, for all $g \in G$. In other words, the associated map $\lambda: \operatorname{Irr}(\mathcal{C}) \rightarrow G$ is surjective. In this case, $\mathcal{C}$ is called a $G$-extension of $\mathcal{C}_{e}$ [12]. When $\mathcal{C}$ is a $G$-extension of a fusion subcategory $\mathcal{D}=\mathcal{C}_{e}$, the FrobeniusPerron dimensions of $\mathcal{C}_{g}$ are all equal and we have $\operatorname{FPdim} \mathcal{C}=|G| \operatorname{FPdim} \mathcal{D}[10$, Proposition 8.20].

Let us recall the notion of nilpotent fusion category from [14]. The adjoint subcategory $\mathcal{C}_{\text {ad }}$ is the full tensor subcategory of $\mathcal{C}$ generated by $X \otimes X^{*}, X \in \operatorname{Irr} \mathcal{C}$. The upper central series $\mathcal{C}=\mathcal{C}^{(0)} \supseteq \mathcal{C}^{(1)} \supseteq \cdots \supseteq \mathcal{C}^{(n)} \supseteq \cdots$ of $\mathcal{C}$ is defined inductively as $\mathcal{C}^{(n)}=\left(\mathcal{C}^{(n-1)}\right)_{\text {ad }}$, for all $n \geq 1$. A fusion category $\mathcal{C}$ is called nilpotent if its upper central series converges to $\operatorname{Vec}_{k}$, that is, if $\mathcal{C}^{(n)}=\operatorname{Vec}_{k}$ for some $n \geq 0$. The smallest such $n$ is called the nilpotency class of $\mathcal{C}$.

For instance, if $p$ is a prime number and $\mathcal{C}$ is any fusion category with $\operatorname{FPdim} \mathcal{C}=p^{n}$, $n \geq 0$, then $\mathcal{C}$ is nilpotent $[14 ; 10]$. 
There is a faithful grading $\mathcal{C}=\bigoplus_{g \in U(\mathcal{C})} \mathcal{C}_{g}$, where $\mathcal{C}_{e}=\mathcal{C}_{\text {ad }}$ and $U(\mathcal{C})$ is the universal grading group of $\mathcal{C}$. Let $\lambda_{\mathcal{C}}: \operatorname{Irr}(\mathcal{C}) \rightarrow U(\mathcal{C})$ denote the universal grading of $\mathcal{C}$. Its universal property can be stated as follows: for any grading $\lambda$ : $\operatorname{Irr}(\mathcal{C}) \rightarrow G$ by a group $G$, there exists a unique group homomorphism $\phi: U(\mathcal{C}) \rightarrow G$ such that $\lambda=\phi \lambda_{\mathcal{C}}$.

Suppose $\mathcal{D}$ is a fusion subcategory of $\mathcal{C}$. Then $\mathcal{D}$ is faithfully graded by the subgroup $U_{\mathcal{D}}(\mathcal{C})=\left\{g \in U(\mathcal{C}) \mid \mathcal{D} \cap \mathcal{C}_{g} \neq 0\right\} \subseteq U(\mathcal{C})$. By the universal property of $U(\mathcal{D})$ there is a surjective group homomorphism $\phi_{\mathcal{D}}: U(\mathcal{D}) \rightarrow U_{\mathcal{D}}(\mathcal{C})$ [14, Corollary 3.7]. For all $g \in U_{\mathcal{D}}(\mathcal{C})$, we have a decomposition $\mathcal{D} \cap \mathcal{C}_{g}=\bigoplus_{\phi_{\mathcal{D}}(t)=g} \mathcal{D}_{t}$.

Let $G$ be a finite group. The category $\mathcal{C}=\operatorname{rep} G$ of finite-dimensional representations of $G$ is nilpotent if and only if $G$ is nilpotent [14]. On the other hand, a fusion subcategory of $\mathcal{C}$ is of the form $\operatorname{rep} G / N$ for some normal subgroup $N$ of $G$. The next theorem amounts in this case to the well-known fact that if $G$ is nilpotent and $N \neq 1$ is a subgroup, then $N \cap Z(G) \neq 1$.

Theorem 2.1 Let $\mathcal{C}$ be a nilpotent fusion category with commutative Grothendieck ring. Suppose $\mathcal{D}$ is a fusion subcategory of $\mathcal{C}$ such that $U_{\mathcal{D}}(\mathcal{C})=U(\mathcal{C})$, that is, $\mathcal{D} \cap \mathcal{C}_{g} \neq 0$, for all $g \in U(\mathcal{C})$. Then $\mathcal{D}=\mathcal{C}$.

The assumption on commutativity of the Grothendieck ring comes from the use, in the proof below, of properties of commutator subcategories, which are proved only under this assumption. See [14, Section 4.2].

Note that the theorem applies, in particular, if $\mathcal{C}$ is braided.

Proof Note first that if $\mathcal{C}^{(1)}=\mathcal{C}_{\text {ad }} \subseteq \mathcal{D}$, then $\mathcal{D}=\mathcal{C}$. Indeed, in this case $\mathcal{D}$ is a $\mathcal{C}_{\text {ad }}-$ sub-bimodule category of $\mathcal{C}$ and therefore, it is a sum of indecomposable sub-bimodule categories $\mathcal{D}=\bigoplus_{g \in S} \mathcal{C}_{g}$, for some subset $S \subseteq U(\mathcal{C})$ (since the subcategories $\mathcal{C}_{g}$ are the indecomposable $\mathcal{C}_{\text {ad }}$-sub-bimodule categories of $\mathcal{C}$ [14]). Hence $\mathcal{D} \cap \mathcal{C}_{g}=0$, for all $g \notin S$, implying, by assumption, that $S=U(\mathcal{C})$, that is, $\mathcal{D}=\bigoplus_{g \in U(\mathcal{C})} \mathcal{C}_{g}=\mathcal{C}$.

Consider the upper central series $\cdots \subseteq \mathcal{C}^{(m+1)} \subseteq \mathcal{C}^{(m)} \subseteq \cdots \subseteq \mathcal{C}^{(1)} \subseteq \mathcal{C}^{(0)}=\mathcal{C}$. Since $\mathcal{C}$ is nilpotent, there exists $n \geq 1$ such that $\mathcal{C}^{(n)}=\operatorname{Vec}_{k}$. In particular, $\mathcal{C}^{(n)} \subseteq \mathcal{D}$. Let $m$ be the smallest nonnegative integer such that $\mathcal{C}^{(m)} \subseteq \mathcal{D}$. We may assume that $m \geq 1$. Then $\mathcal{C}^{(m-1)} \nsubseteq \mathcal{D}$ and $\mathcal{C}^{(m)}=\left(\mathcal{C}^{(m-1)}\right)_{\text {ad }} \subseteq \mathcal{D}$.

Let $\mathcal{D}^{\text {co }}$ be commutator subcategory of $\mathcal{D}$ in $\mathcal{C}$, that is, $\mathcal{D}^{\text {co }}$ is the fusion subcategory of $\mathcal{C}$ generated by all simple objects $Y$ of $\mathcal{C}$ such that $Y \otimes Y^{*}$ belongs to $\mathcal{D}$ [14, Definition 4.10]. Thus $\left(\mathcal{D}^{\text {co }}\right)_{\text {ad }} \subseteq \mathcal{D} \subseteq \mathcal{D}^{\text {co }}$, and $\mathcal{D}^{\text {co }}$ is the largest fusion subcategory of $\mathcal{C}$ with the property $\left(\mathcal{D}^{\text {co }}\right)_{\text {ad }} \subseteq \mathcal{D}$. 
For every $j=0, \ldots$, let $\mathcal{D}^{[j]}$ be the fusion subcategory defined as follows: $\mathcal{D}^{[0]}=\mathcal{D}$, $\mathcal{D}^{[j+1]}=\left(\mathcal{D}^{[j]}\right)^{\mathrm{co}}, j \geq 0$. As before, we have $\left(\mathcal{D}^{[j+1]}\right)_{\text {ad }} \subseteq \mathcal{D}^{[j]} \subseteq \mathcal{D}^{[j+1]}$, for all $j=0,1, \ldots$.

Since $\mathcal{D} \subseteq \mathcal{D}^{[j]}$, we get:

$$
\mathcal{D}^{[j]} \cap \mathcal{C}_{g} \neq 0, \quad \forall g \in U(\mathcal{C}), \forall j=0, \ldots
$$

On the other hand, we have inclusions

$$
\mathcal{C}^{(m-j)} \subseteq \mathcal{D}^{[j]}, \quad \forall j=0, \ldots, m .
$$

Indeed, $\mathcal{C}^{(m)}=\left(\mathcal{C}^{(m-1)}\right)_{\text {ad }} \subseteq \mathcal{D}$, so that $\mathcal{C}^{(m-1)} \subseteq \mathcal{D}^{\mathrm{co}}=\mathcal{D}^{[1]}$. Assuming inductively that $\mathcal{C}^{(m-j)} \subseteq \mathcal{D}^{[j]}$ for $1 \leq j<m$, we get that $\left(\mathcal{C}^{(m-(j+1))}\right)$ ad $=\mathcal{C}^{(m-j)} \subseteq \mathcal{D}^{[j]}$. Hence $\mathcal{C}^{(m-(j+1))} \subseteq\left(\mathcal{D}^{[j]}\right)^{\mathrm{co}}=\mathcal{D}^{[j+1]}$.

It follows from (3) that $\mathcal{C}^{(1)}=\mathcal{C}_{\mathrm{ad}} \subseteq \mathcal{D}^{[m-1]}$. Therefore $\mathcal{D}^{[m-1]}=\mathcal{C}$, in view of (2). We now show by induction that $\mathcal{D}^{[m-j]}=\mathcal{C}$, for all $j=1, \ldots, m$. Suppose that $\mathcal{D}^{[m-j]}=\mathcal{C}$. Then $\mathcal{C}_{\text {ad }}=\left(\mathcal{D}^{[m-j]}\right)$ ad $\subseteq \mathcal{D}^{[m-(j+1)]}$. Hence $\mathcal{D}^{[m-(j+1)]}=\mathcal{C}$. In particular, $\mathcal{D}=\mathcal{D}^{[0]}=\mathcal{C}$. This finishes the proof of the theorem.

\section{The fusion subcategory generated by a simple object}

Let $\mathcal{C}$ be a fusion category. Recall that an object $X$ of $\mathcal{C}$ is called faithful if for every $Y \in \operatorname{Irr}(\mathcal{C})$, we have $m\left(Y, X^{\otimes n}\right)>0$, for some integer $n \geq 1$. Thus $X \in \mathcal{C}$ is faithful if and only if $\{X\}$ generates $\mathcal{C}$ as a fusion category.

The following definition appears in [17, Chapter 4].

Definition 3.1 Let $X$ be a nonzero object of $\mathcal{C}$. The smallest nonnegative integer $n$ such that $m\left(\mathbb{1}, X^{\otimes n}\right)>0$ is called the $\operatorname{order}$ of $X$. We shall denote it by $\operatorname{ord}(X)$.

Suppose $X \in \operatorname{Irr}(\mathcal{C})$. Then $\operatorname{ord}(X)=1$ if and only if $X=\mathbb{1}$. Also, $\operatorname{ord}(X)=2$ if and only if $X=X^{*}$ and $\mathbb{1} \neq X$.

Remark Recall that the rank of $\mathcal{C}$, denoted $\operatorname{rk}(\mathcal{C})$, is the cardinality of the $\operatorname{set} \operatorname{Irr}(\mathcal{C})$. It is shown in [17, Proposition 5.1] that an object $X$ is faithful if and only if the matrix of left multiplication by $X$ in the basis $\operatorname{Irr}(\mathcal{C})$ of the Grothendieck ring of $\mathcal{C}$ is indecomposable. As in [17, Corollary 5.1], we get that $\operatorname{ord}(X) \leq \operatorname{rk}(\mathcal{C})$, for all nonzero object $X$. Indeed, these results are stated [loc. cit.] for the case where $\mathcal{C}$ is the category of finite-dimensional representations of a semisimple Hopf algebra, but the proof applies mutatis mutandis in any fusion category as well. 
Suppose that $\mathcal{C}$ is a spherical fusion category. Then, by $\mathrm{Ng}$ and Schauenburg [22, Corollary 5.13], for every simple object $X$ of $\mathcal{C}$, we have furthermore that $\operatorname{ord}(X) \leq$ FSexp $(\mathcal{C})$, where $\mathrm{FSexp}(\mathcal{C}) \in \mathbb{N}$ is the Frobenius-Schur exponent of $\mathcal{C}$.

Let $X$ be an object of $\mathcal{C}$ and let $\mathcal{C}[X]$ be the fusion subcategory of $\mathcal{C}$ generated by $X$. The universal grading group of $\mathcal{C}[X]$ will be denoted by $U(X)$.

Remark Let $\mathcal{C}$ be a fusion category and assume $\mathcal{C}$ has a unique maximal fusion subcategory. Then $\mathcal{C}$ has a faithful simple object.

Proof Let $\mathcal{D} \subsetneq \mathcal{C}$ be the unique maximal fusion subcategory of $\mathcal{C}$. If $X \in \operatorname{Irr}(\mathcal{C})$ is such that $\mathcal{C}[X] \subsetneq \mathcal{C}$, then $\mathcal{C}[X] \subseteq \mathcal{D}$. Hence there must exist some $X \in \operatorname{Irr}(\mathcal{C})$ with $\mathcal{C}[X]=\mathcal{C}$. This proves the claim.

Example 3.2 (i) Let $\mathcal{C}$ be a pointed fusion category with a finite group $G$ of invertible objects. Then a simple object $g \in G$ is faithful if and only if $G$ is a cyclic group generated by $g$.

(ii) Suppose $\mathcal{C}=\operatorname{rep} G$, where $G$ a finite group. Let $X$ be an object of $\mathcal{C}$, and let $\chi=\chi_{X}$ be the character of $X$. Then $X$ is a faithful object of $\mathcal{C}$ if and only if $\chi$ is a faithful character of $G$.

We have in this example $\mathcal{C}[X]=\operatorname{rep}(G / \operatorname{ker} \chi)$. Moreover, if $X \in \operatorname{Irr}(\mathcal{C})$ (that is, $X$ is an irreducible representation of $G)$, then $U(X) \simeq Z(G / \operatorname{ker} \chi) \simeq Z(\chi) / \operatorname{ker} \chi[16$, Lemma 2.27]. Recall that the normal subgroups $\operatorname{ker} \chi$ and $Z(\chi)$ are defined as:

(4) $\operatorname{ker} \chi=\{g \in G \mid \chi(g)=\chi(1)\}, \quad Z(\chi)=\left\{g \in G|| \chi(g) \mid=\left(\chi \chi^{*}\right)(g)=\chi(1)\right\}$

(iii) Let $H$ be a semisimple Hopf algebra and let $\mathcal{C}=$ rep $H$ be the category of finite-dimensional representations of $H$. Let $\pi: H \rightarrow \operatorname{End}(V)$ be a finite-dimensional representation. The subspaces

$$
\begin{aligned}
\operatorname{Rker}(\pi) & =\left\{h \in H \mid \pi\left(h_{(1)}\right) \otimes h_{(2)}=\mathrm{id}_{V} \otimes h\right\}, \\
\operatorname{RZ}(\pi) & =\operatorname{Rker}\left(\pi \otimes \pi^{*}\right)=\left\{h \in H \mid\left(\pi \otimes \pi^{*}\right)\left(h_{(1)}\right) \otimes h_{(2)}=\mathrm{id}_{V \otimes V^{*}} \otimes h\right\},
\end{aligned}
$$

are normal right coideal subalgebras of $H$. (Similarly, one can define normal left coideal subalgebras $\operatorname{Lker}(\pi)$ and $\operatorname{LZ}(\pi)$ with analogous properties.) The coideal subalgebras $\operatorname{Rker}(\pi)$ and $\operatorname{Lker}(\pi)$ are studied in Burciu [7].

The quotient Hopf algebra $H / H(\operatorname{Rker} \pi)^{+}$satisfies $\mathcal{C}[\pi]=\operatorname{rep}\left(H / H(\operatorname{Rker} \pi)^{+}\right)$. We have in addition: 
Proposition 3.3 Let $A \subseteq H$ be a right coideal subalgebra. Then $A \subseteq \mathrm{RZ}(\pi)$ if and only if, for all $a \in A, \pi(a)=\lambda(a) \mathrm{id}_{V}$, for some linear character $\lambda \in G\left(A^{*}\right)$. In particular, if $A \subseteq \mathrm{RZ}(\pi)$ and $\pi$ is faithful then $A \subseteq Z(H)$.

Proof Note that if an element $h \in H$ belongs to $\operatorname{RZ}(\pi)=\operatorname{Rker}\left(\pi \otimes \pi^{*}\right)$, then $h$ acts trivially on $V \otimes V^{*}$. Since $V \otimes V^{*} \simeq \operatorname{End}(V)$ as $H$-modules, this implies that there exists $\lambda(h) \in k$ such that $\pi(h)=\lambda(h) \mathrm{id}_{V}$. In other words, the normal right coideal subalgebra $\operatorname{RZ}(\pi)$ acts by scalars on $V$. Clearly, the map $\lambda$ defines a linear character on $\operatorname{RZ}(\pi)$. This implies the only if direction.

Conversely, suppose that for all $a \in A, \pi(a)=\lambda(a) \mathrm{id}_{V}$, for some linear character $\lambda \in G\left(A^{*}\right)$. Then $A$ acts trivially on $\operatorname{End}(V) \simeq V \otimes V^{*}$ and since $A$ is a right coideal subalgebra, we get that $A \subseteq \mathrm{RZ}(\pi)$. This finishes the proof of the lemma.

Let $K \subseteq H$ be the maximal central Hopf subalgebra. It follows from [14, Theorem 3.8] that $K \simeq k^{U(H)}$, where $U(H)$ is the universal grading group of $\mathcal{C}=$ rep $H$. Thus, $U(H)$ is isomorphic to the group $G\left(K^{*}\right)$ of one-dimensional representations of $K$.

The following corollary describes the universal grading group $U(H)$, generalizing [16, Corollary 2.28]. The author thanks S Burciu for his comments on a previous version of the manuscript that motivated the present statement.

Corollary 3.4 Let $H$ be a semisimple Hopf algebra and let $U(H)$ be the universal grading group of the category rep $H$. Then $k^{U(H)}=\bigcap_{\pi \in \operatorname{Irr}(H)} \mathrm{RZ}(\pi)$.

Proof Let $R=\bigcap_{\pi \in \operatorname{Irr}(H)} \mathrm{RZ}(\pi)$. As remarked above, we have an isomorphism $k^{U(H)} \simeq K$, where $K$ is the maximal central Hopf subalgebra of $H$. Since $K \subseteq Z(H)$, we have $K \subseteq R$, by Proposition 3.3.

Note that $H / H K^{+}=H_{\text {ad }}$ is the unique quotient Hopf algebra that satisfies rep $H_{\text {ad }}=$ $(\text { rep } H)_{\text {ad }}$. Let $p: H \rightarrow H_{\text {ad }}$ denote the canonical projection, so that $K={ }^{\operatorname{co} p} H=$ $\left\{h \in H: p\left(h_{(1)}\right) \otimes h_{(2)}=1 \otimes h\right\}$.

Since $R$ is a central right coideal subalgebra, there is a Hopf algebra quotient $p^{\prime}: H \rightarrow$ $H / H R^{+}$and we have as well $R={ }^{\operatorname{co} p^{\prime}} H=\left\{h \in H: p^{\prime}\left(h_{(1)}\right) \otimes h_{(2)}=1 \otimes h\right\}$. Because elements of $R$ act trivially on the tensor products $V \otimes V^{*}$, for all simple $H$-modules $V$, it follows that $R$ acts trivially on every irreducible representation of $H_{\text {ad }}$. Hence $\left.p\right|_{R}=\epsilon$ and thus $H R^{+} \subseteq \operatorname{ker} p=H K^{+}$. Then the projection $p$ factorizes through $p^{\prime}: H \rightarrow H / H R^{+}$, that is, $p=f p^{\prime}$, where $f: H / H R^{+} \rightarrow H / H K^{+}$is a Hopf algebra map. Therefore $R={ }^{\text {co } p^{\prime}} H \subseteq{ }^{\text {co } p} H=K$. This implies that $R=K$ and finishes the proof of the corollary. 
Let $\mathcal{C}$ be a fusion category and let $X \in \operatorname{Irr}(\mathcal{C})$. Recall that by the universal property of $U(X)$ there is a group homomorphism $\phi_{X}=\phi_{\mathcal{C}[X]}: U(X) \rightarrow U(\mathcal{C})$. This is determined as follows: for every $t \in U(X), \phi_{X}(t)=g \in U(\mathcal{C})$ if and only if $\mathcal{C}[X]_{t} \subseteq \mathcal{C}_{g}$. The following lemma will be used later on.

Lemma 3.5 We have $U(\mathcal{C})=\bigcup_{X \in \operatorname{Irr}(\mathcal{C})} \phi_{X}(U(X))$.

Proof Let $g \in U(\mathcal{C})$ and let $Y$ be a simple object in $\mathcal{C}_{g}$. By definition of $\phi_{Y}$, we have $g \in \phi_{Y}(U(Y))$. This proves the lemma.

We end this section by recalling some known families of examples. Let $k=\mathbb{C}$.

Example 3.6 (Verlinde categories for $\mathfrak{s l}_{2}$ ) Let $n$ be a positive integer and let $q=$ $e^{i \pi /(n+2)}$. Let $\mathcal{C}_{n}=\mathcal{C}_{n}\left(\mathfrak{s l}_{2}\right)$ be the semisimplification of the category of representations of $U_{q}\left(\mathfrak{s l}_{2}\right)$ (Andersen and Paradowski [1], and Bakalov and Kirillov [2]). It is wellknown that $\mathcal{C}_{n}$ is a modular fusion category over $k$. Isomorphism classes of simple objects in $\mathcal{C}_{n}$ are represented by objects $X_{i}, 0 \leq i \leq n$, with $X_{0}=\mathbb{1}, X_{i}^{*}=X_{i}$, and obeying the truncated Clebsch-Gordan fusion rules:

$$
X_{i} \otimes X_{j} \simeq \bigoplus_{\substack{l=|i-j| \\ i+j \equiv l(2)}}^{\min (i+j, 2 n-(i+j))} X_{l}
$$

The Frobenius-Perron dimension of $X_{j}$ is given by

$$
\operatorname{FPdim} X_{j}=\frac{q^{j+1}-q^{-(j+1)}}{q-q^{-1}}=\frac{\sin ((j+1) \theta)}{\sin (\theta)}, \quad \text { where } \theta=\frac{\pi}{n+2} .
$$

In particular, there are exactly two invertible objects: $\mathbb{1}=X_{0}$ and $g=X_{n}$.

There is a faithful $\mathbb{Z}_{2}$-grading on $\mathcal{C}_{n}$ given by $\mathcal{C}_{n}=\mathcal{C}_{n}^{+} \oplus \mathcal{C}_{n}^{-}$, where $\mathcal{C}_{n}^{ \pm}$is the full Abelian subcategory with simple objects $X_{i}, i$ even (respectively, odd). Letting $X=X_{1}$, relation (5) implies that $\mathcal{C}_{n}=\mathcal{C}_{n}[X]$, so that $X$ is a faithful simple object of order 2 . We have in this example $U\left(\mathcal{C}_{n}\right) \simeq G\left(\mathcal{C}_{n}\right) \simeq \mathbb{Z}_{2}$.

Example 3.7 (Modular near-group categories) Let $\mathcal{C}$ be a near-group category of type $(G, \kappa)$, as described in the introduction. Then we have $U(\mathcal{C})=1$ if $\kappa>0$, and $U(\mathcal{C})=\mathbb{Z}_{2}$ if $\kappa=0$. Indeed, in the first case, it is clear from (1) that $\mathcal{C}_{\text {ad }}=\mathcal{C}$, while in the second case $\mathcal{C}_{\text {ad }} \simeq \mathcal{C}[G]$.

Let $\mathcal{C}$ be a near-group category of type $(G, \kappa)$ and suppose that $\mathcal{C}$ admits a modular structure. Then $G=G(\mathcal{C}) \simeq U(\mathcal{C})$ is of order 1 or 2 . 
If $\kappa \neq 0$, then $G=1$, so $\mathcal{C}$ is of rank 2 and has a non-invertible object $X$, such that $X^{\otimes 2}=\mathbb{1} \oplus X$. By Ostrik [23] there are 4 nonequivalent braided fusion categories with these fusion rules, called Yang-Lee categories, and they are modular.

If $\kappa=0$, then $G \simeq \mathbb{Z}_{2}$. The fusion rule (1) is in this case $X^{\otimes 2}=\mathbb{1} \oplus a$, where $\langle a\rangle=G$. Fusion categories with these fusion rules are called Ising categories. They are classified in [9, Appendix B]. In particular, every braided Ising category is modular [9, Corollary B.12].

Example 3.8 (Fermionic Moore-Read fusion rule) Let $G=\left\langle g \mid g^{4}=e\right\rangle$ denote the cyclic group of order 4 . Consider the commutative fusion rules on the set $G \cup\left\{X, X^{\prime}\right\}$ determined by $h \otimes h^{\prime} \simeq h h^{\prime}, h, h^{\prime} \in G$ and

$$
\begin{aligned}
& g^{2} \otimes X \simeq X, \quad g^{2} \otimes X^{\prime} \simeq X^{\prime}, \quad X \otimes X^{\prime} \simeq \mathbb{1} \oplus g^{2}, \\
& g \otimes X \simeq X^{\prime}, \quad g \otimes X^{\prime} \simeq X, \quad X \otimes X \simeq g \oplus g^{3}, \\
& g^{3} \otimes X \simeq X^{\prime}, \quad g^{3} \otimes X^{\prime} \simeq X, \quad X^{\prime} \otimes X^{\prime} \simeq g \oplus g^{3} .
\end{aligned}
$$

It is known that, up to equivalence of tensor categories, there are four fusion categories with these fusion rules, none of them braided (Bonderson [4], and Liptrap [18]). If $\mathcal{C}$ is any fusion category satisfying these fusion rules, then $\operatorname{FPdim} \mathcal{C}=8$ and $\mathcal{C}$ is nilpotent of nilpotency class 2 . We have in addition $G=G(\mathcal{C}) \simeq U(\mathcal{C})$ and $\mathcal{C}_{\text {ad }}=\mathcal{C}\left[g^{2}\right]$.

Observe that in these examples $g^{2}$ is the only simple object of order 2. Also, $X$ is of order 4 and $X^{\otimes 2}$ decomposes as a direct sum of simple objects of order 4 .

Example 3.9 (Faithful simple objects of order 2) Let $\mathcal{C}$ be a fusion category over $k$ and assume $X$ is a faithful simple object of $\mathcal{C}$ of order 2, that is, $\mathcal{C}=\mathcal{C}[X], X \neq \mathbb{1}$ and $X \simeq X^{*}$.

Let $q \in k^{\times}$and consider the tensor category $\operatorname{rep} \mathrm{SL}_{q}(2)$ of finite-dimensional comodules over the Hopf algebra $\mathrm{SL}_{q}(2)$. Suppose $q$ is generic, that is, it is not a root of unity or $q= \pm 1$. Then $\operatorname{rep} \operatorname{SL}_{q}(2)$ is a semisimple tensor category whose Grothendieck ring is isomorphic to the Grothendieck ring of the category rep SL(2). The category rep $\mathrm{SL}_{q}(2)$ has a self-dual faithful simple object $V$ corresponding to the standard 2-dimensional representation of SL(2).

Fix an isomorphism $\Phi: X \rightarrow X^{*}$ such that the induced map

$$
\mathbb{1} \rightarrow X \otimes X^{*} \stackrel{\Phi \otimes \Phi^{-1}}{\longrightarrow} X^{*} \otimes X \rightarrow \mathbb{1}
$$

is given by the scalar $-\left(q+q^{-1}\right)$, where $q \in k^{\times}$is generic. In this case there exists a unique tensor functor $F: \operatorname{repSL} q(2) \rightarrow \mathcal{C}$ such that $F(V)=X$ and $F(\phi)=\Phi$, 
where $\phi: V \rightarrow V^{*}$ is a fixed isomorphism in rep $\mathrm{SL}_{q}(2)$. See Etingof and Ostrik [13, Theorem 2.1], and Turaev [30, Chapter XII]. Since $X$ is a faithful object and $F(V)=X$, the functor $F$ is surjective. Thus, every such fusion category $\mathcal{C}$ is a quotient of $\operatorname{rep} \mathrm{SL}_{q}(2)$ for an appropriate value of $q$. (Note that when $q$ is a root of unity, the category $\operatorname{rep~} \mathrm{SL}_{q}(2)$ has a similar universal property [13, Theorem 2.3].)

Example 3.10 (Faithful comodules of dimension 2) Finite-dimensional cosemisimple Hopf algebras with a self-dual faithful irreducible comodule $V$ of dimension 2 were classified in Bichon and Natale [3].

Let $v(V)= \pm 1$ denote the Frobenius-Schur indicator of $\underset{\widetilde{\Omega}}{V}$. If $v(V)=-1$, then $H$ is commutative and isomorphic to the dual group algebra $k^{\widetilde{\Gamma}}$, where $\widetilde{\Gamma}$ is a non-Abelian binary polyhedral group.

If, on the other hand, $v(V)=1$, then either $H$ is commutative and isomorphic to $k^{D_{n}}, n \geq 3$, where $D_{n}$ is the dihedral group of order $2 n$, or $H$ is isomorphic to one of certain nontrivial Hopf algebra deformations $\mathcal{A}[\tilde{\Gamma}]$ or $\mathcal{B}[\tilde{\Gamma}]$ of a binary polyhedral group $\widetilde{\Gamma}$. In the last case, $H$ fits into an Abelian cocentral exact sequence $k \rightarrow k^{\Gamma} \rightarrow H \rightarrow k \mathbb{Z}_{2} \rightarrow k$, where $\widetilde{\Gamma} / \mathbb{Z}_{2}=\Gamma \subseteq \mathrm{PSL}_{2}(k)$ is a finite polyhedral group of even order. The universal grading group of the category $\mathcal{C}$ of finite-dimensional $H$-comodules is $\mathbb{Z}_{2}$, and the adjoint subcategory is the category of representations of the commutative Hopf subalgebra $k^{\Gamma}$.

\section{Faithful simple objects and the universal grading group}

Let $G$ be a finite group and let $\mathcal{C}=\operatorname{rep} G$. Then $U(\mathcal{C}) \simeq Z(G)$. A classical result says that if $G$ has a faithful character, then the center $Z(G)$ is cyclic; see for instance [16, Theorem 2.32 (a)]. The analogous statement is true for any fusion category, as follows from the next theorem.

Theorem 4.1 Let $\mathcal{C}$ be a fusion category and let $U(\mathcal{C})$ be its universal grading group. Then the following hold:

(i) Suppose $X \in \operatorname{Irr}(\mathcal{C})$ and let $g \in U(\mathcal{C})$ such that $X \in \mathcal{C}_{g}$. Then the order of $g$ divides the order of $X$.

(ii) Suppose $\mathcal{C}$ is generated by simple objects $X_{1}, \ldots, X_{m}$ as a fusion category, and let $g_{i} \in U(\mathcal{C})$ such that $X_{i} \in \mathcal{C}_{g_{i}}, 1 \leq i \leq m$. Then $g_{1}, \ldots, g_{m}$ generate the group $U(\mathcal{C})$.

In particular, if $X \in \operatorname{Irr}(\mathcal{C})$ is faithful, then the group $U(\mathcal{C})$ is cyclic and its order divides the order of $X$. 
As a consequence, if $\mathcal{C}$ has a self-dual faithful simple object, then $U(\mathcal{C})=1$ or $U(\mathcal{C}) \simeq \mathbb{Z}_{2}$

Proof (i) Let $n=\operatorname{ord}(X)$, so that $m\left(\mathbb{1}, X^{\otimes n}\right)>0$. On the other hand, $X^{\otimes n} \in \mathcal{C}_{g^{n}}$, and since $\mathbb{1} \in \mathcal{C}_{e}$, we get $\mathcal{C}_{g^{n}}=\mathcal{C}_{e}$. Hence $g^{n}=e$, and the order of $g$ divides $n$.

(ii) Since $X_{i} \in \mathcal{C}_{g_{i}}, 1 \leq i \leq m$, then $X_{i_{1}} \otimes \cdots \otimes X_{i_{t}} \in \mathcal{C}_{g_{i_{1}} \cdots g_{i_{t}}}$, for all $1 \leq i_{1}, \ldots, i_{t} \leq$ $m$. Let $g \in U(\mathcal{C})$ and let $Y \in \operatorname{Irr}(\mathcal{C})$ such that $Y \in \mathcal{C}_{g}$. By assumption, $Y$ appears with positive multiplicity in some tensor product $X_{i_{1}} \otimes \cdots \otimes X_{i_{t}}$. Then $Y \in \mathcal{C}_{g_{i_{1}} \cdots g_{i_{t}}}$ and we get that $g=g_{i_{1}} \cdots g_{i_{t}}$. Thus $U(\mathcal{C})=\left\langle g_{i_{1}}, \ldots, g_{i_{t}}\right\rangle$, as claimed.

Example 4.2 Let $H$ be a semisimple Hopf algebra and let $K \subseteq H$ be the maximal central Hopf subalgebra of $H$, so that $K \simeq k^{U(\mathcal{C})}$, where $\mathcal{C}=\operatorname{rep} H$ is the fusion category of finite dimensional representations of $H$. Theorem 4.1 implies that if $H$ has a faithful irreducible character $\chi$, then $K \simeq k^{\mathbb{Z}_{m}}$, where $m$ divides the order of $\chi$. (Compare with [3, Theorem 3.5].)

Corollary 4.3 Let $p$ be a prime number. Suppose $\mathcal{C}$ is a nilpotent fusion category such that $\mathcal{C}$ has a simple object of order $p$. Then the Frobenius-Perron dimension of $\mathcal{C}$ is divisible by $p$. In particular, if $\mathcal{C}$ has a self-dual simple object, then FPdim $\mathcal{C}$ is even.

Note that the Frobenius-Perron dimension of a nilpotent fusion category is always an integer.

Proof Let $X \in \operatorname{Irr}(\mathcal{C})$ of order $p$. Since $\mathcal{C}[X]$ is also nilpotent, then $U(X) \neq 1$ and therefore $U(X) \simeq \mathbb{Z}_{p}$, by Theorem 4.1. Hence $p$ divides FPdim $\mathcal{C}[X]$. This implies the corollary, since $\mathrm{FPdim} \mathcal{C}[X] \operatorname{divides} \operatorname{FPdim} \mathcal{C}$ [10, Proposition 8.15].

Combining Lemma 3.5 and Theorem 4.1 we get the following:

Corollary 4.4 Let $\mathcal{C}$ be a fusion category. Then the following hold:

(i) Let $n \in \mathbb{N}$ and suppose that the order of $X$ divides $n$, for all $X \in \operatorname{Irr} \mathcal{C}$. Then the exponent of $U(\mathcal{C})$ divides $n$. In particular, if all simple objects of $\mathcal{C}$ are self-dual, then $U(\mathcal{C})$ is an elementary Abelian 2-group.

(ii) Let $p$ be a prime number. Suppose that $\mathcal{C}$ is nilpotent and the order of $X$ is a power of $p$, for all simple object $X$ of $\mathcal{C}$. Then $\operatorname{FPdim} \mathcal{C}=p^{m}$, for some $m \geq 1$. 
Proof By Theorem 4.1 each of the groups $U(X)$ is cyclic of order dividing $n$. Thus (i) follows from Lemma 3.5. To prove (ii), observe that it follows from Lemma 3.5 and Theorem 4.1 that $U(\mathcal{C})$ is a $p$-group, hence of order a power of $p$. Since $\mathcal{C}$ is nilpotent, $F \operatorname{Fdim} \mathcal{C}_{\text {ad }}<\operatorname{FPdim} \mathcal{C}$. By induction, we may assume that $\operatorname{FPdim} \mathcal{C}_{\text {ad }}$ is a power of $p$. Then so is $\mathrm{FPdim} \mathcal{C}$.

The next lemma gives a sufficient condition for a fusion category $\mathcal{C}$ to have a self-dual simple object, in terms of the universal grading of $\mathcal{C}$.

Lemma 4.5 Suppose that $g \in U(\mathcal{C})$ is of order 2 and the rank of $\mathcal{C}_{g}$ is 1 . Then, if $X \in \mathcal{C}_{g}$ is a simple object of $\mathcal{C}, X$ has order 2 .

Proof Let $X \in \mathcal{C}_{g}$. Then $X^{*} \in \mathcal{C}_{g^{-1}}=\mathcal{C}_{g}$, because $g$ is of order 2, by assumption. Since $\mathcal{C}_{g}$ has rank 1 , then $X^{*}$ must be isomorphic to $X$. This implies the lemma.

Recall that if $G$ is a finite nilpotent group with cyclic center, then $G$ has a faithful irreducible character [16, Theorem 2.32(b) and Problem 4.3]. Our next theorem establishes the analogous fact for braided fusion categories, thus giving a partial converse of Theorem 4.1 in this case.

We need first the following lemma.

Lemma 4.6 Let $\mathcal{C}_{1}, \mathcal{C}_{2}$ be nilpotent fusion categories with commutative Grothendieck ring. Suppose that $X_{i}$ is a faithful simple object of $\mathcal{C}_{i}, i=1,2$. Assume in addition that the orders of $U\left(\mathcal{C}_{1}\right)$ and $U\left(\mathcal{C}_{2}\right)$ are relatively prime. Then $X_{1} \otimes X_{2}$ is a faithful simple object of $\mathcal{C}_{1} \otimes \mathcal{C}_{2}$.

Proof Let $\mathcal{C}=\mathcal{C}_{1} \otimes \mathcal{C}_{2}$ and put $U_{i}=U\left(\mathcal{C}_{i}\right)$ and $U=U(\mathcal{C})$. Note that $\mathcal{C}_{\text {ad }}=$ $\left(\mathcal{C}_{1}\right)_{\mathrm{ad}} \otimes\left(\mathcal{C}_{2}\right)_{\text {ad }}$ and $U=U_{1} \times U_{2}$. In particular, $\mathcal{C}$ is also nilpotent.

By Theorem 4.1 the groups $U_{1}$ and $U_{2}$ are cyclic. Since $\left|U_{1}\right|$ and $\left|U_{2}\right|$ are relatively prime, then $U$ is also cyclic. Moreover, suppose that $X_{i} \in\left(\mathcal{C}_{i}\right)_{a_{i}}, i=1,2$. Then $\left\langle a_{i}\right\rangle=U_{i}$ and thus $U=\langle a\rangle$, where $a=\left(a_{1}, a_{2}\right)$.

Let $g \in U, g=a^{m}=\left(a_{1}^{m}, a_{2}^{m}\right), m \geq 1$. Then $\left(X_{1} \otimes X_{2}\right)^{\otimes m}=X_{1}^{\otimes m} \otimes X_{2}^{\otimes m}$ is a nonzero object in $\left(\mathcal{C}_{1}\right)_{a_{1}^{m}} \otimes\left(\mathcal{C}_{2}\right)_{a_{2}^{m}}=\left(\mathcal{C}_{1} \otimes \mathcal{C}_{2}\right)_{\left(a_{1}^{m}, a_{2}^{m}\right)}=\mathcal{C}_{g}$.

Denote by $\mathcal{D}=\mathcal{C}\left[X_{1} \otimes X_{2}\right]$ the fusion subcategory of $\mathcal{C}$ generated by $X_{1} \otimes X_{2}$. We have shown that $\mathcal{D} \cap \mathcal{C}_{g} \neq 0$, for all $g \in U(\mathcal{C})$. Since $\mathcal{C}$ is also a nilpotent fusion category with commutative Grothendieck ring, $\mathcal{D}=\mathcal{C}$, by Theorem 2.1.

Theorem 4.7 Let $\mathcal{C}$ be a braided nilpotent fusion category such that the group $U(\mathcal{C})$ is cyclic. Then $\mathcal{C}$ has a faithful simple object. 
Proof Suppose first that $\operatorname{FPdim} \mathcal{C}=p^{n}$, where $p$ is a prime number, $n \geq 0$. By assumption, $U(\mathcal{C})$ has a unique subgroup $T$ of index $p$. Let $X \in \operatorname{Irr}(\mathcal{C})$ and suppose that $\mathcal{C}[X] \subsetneq \mathcal{C}$. It follows from Theorem 2.1 that $\phi_{X}(U(X)) \subsetneq U(\mathcal{C})$ and therefore $\phi_{X}(U(X)) \subseteq T$. By Lemma 3.5, $U(\mathcal{C})=\bigcup_{X \in \operatorname{Irr}(\mathcal{C})} \phi_{X}(U(X))$. Therefore there must exist some $X \in \operatorname{Irr}(\mathcal{C})$ with $\mathcal{C}[X]=\mathcal{C}$. Then the theorem holds in this case.

By [8, Theorem 1.1] a braided nilpotent fusion category has a unique decomposition into a tensor product of braided fusion categories whose Frobenius-Perron dimensions are powers of distinct primes. That is, there exist prime numbers $p_{1}, \ldots, p_{r}, p_{i} \neq p_{j}$ for all $i \neq j$, and an equivalence of braided fusion categories $\mathcal{C} \simeq \mathcal{C}_{p_{1}} \otimes \cdots \otimes \mathcal{C}_{p_{r}}$, where for all $i=1, \ldots, r, \mathcal{C}_{p_{i}}$ is a braided fusion category of Frobenius-Perron dimension $p_{i}^{n_{i}}$, for some $n_{i} \geq 0$.

We have an isomorphism $U(\mathcal{C}) \simeq U\left(\mathcal{C}_{p_{1}}\right) \times \cdots \times U\left(\mathcal{C}_{p_{r}}\right)$. Therefore the groups $U\left(\mathcal{C}_{p_{i}}\right)$ are also cyclic. As we have already shown, this implies that each $\mathcal{C}_{p_{i}}$ has a faithful simple object $X_{i}, i=1, \ldots, r$. Since the orders of the groups $U\left(\mathcal{C}_{p_{i}}\right)$ are relatively prime, Lemma 4.6 implies that $X_{1} \otimes \cdots \otimes X_{r}$ is a faithful simple object of $\mathcal{C}$. This finishes the proof of the theorem.

When $\mathcal{C}$ is a braided fusion category, the group $U(\mathcal{C})$ is Abelian. We have in this case the following refinement of Corollary 4.4:

Proposition 4.8 Let $\mathcal{C}$ be a braided fusion category. Suppose $\mathcal{C}$ is generated by simple objects $X_{1}, \ldots, X_{n}$. Then the exponent of $U(\mathcal{C})$ divides 1.c.m. $\left\{\right.$ ord $\left.X_{i} \mid 1 \leq i \leq n\right\}$. In particular, if $\mathcal{C}$ is generated by self-dual simple objects, then $U(\mathcal{C})$ is an elementary Abelian 2-group.

Proof Let $g_{i} \in U(\mathcal{C})$ such that $X_{i} \in \mathcal{C}_{g_{i}}$. Then $U(\mathcal{C})$ is generated by $g_{1}, \ldots, g_{n}$, and by Theorem 4.1, the order of $g_{i}$ divides ord $X_{i}$, for all $i=1, \ldots, n$. This implies the proposition, since $U(\mathcal{C})$ is Abelian.

When $\mathcal{C}$ is a modular category, there is a group isomorphism $G(\mathcal{C}) \simeq U(\mathcal{C})$ [14]. Theorems 4.1 and 4.7 imply the following:

Corollary 4.9 Let $\mathcal{C}$ be a modular category. Then the following hold:

(i) Suppose $\mathcal{C}$ has a faithful simple object. Then the group $G(\mathcal{C})$ is cyclic and its order divides the order of $X$. In particular, if $\mathcal{C}$ has a faithful self-dual simple object, then $G(\mathcal{C})=1$ or $G(\mathcal{C}) \simeq \mathbb{Z}_{2}$.

(ii) Suppose $\mathcal{C}$ is nilpotent. If $G(\mathcal{C})$ is cyclic, then $\mathcal{C}$ has a faithful simple object. 


\section{Generalized Tambara-Yamagami fusion rules}

Let us consider a fusion category $\mathcal{C}$ such that $\mathcal{C}$ is not pointed and for all non-invertible simple objects $X, Y$ of $\mathcal{C}$, their tensor product $X \otimes Y$ is a direct sum of invertible objects. We shall say in this case that $\mathcal{C}$ has generalized Tambara-Yamagami fusion rules. These categories are classified, up to equivalence of tensor categories, in [18]. Semisimple Hopf algebras $H$ such that the category rep $H$ has generalized TambaraYamagami fusion rules were studied in Natale [21].

Let $G$ be the group of invertible objects of $\mathcal{C}$. Then for all $X \in \operatorname{Irr}(\mathcal{C})$ we have the relation $X \otimes X^{*} \simeq \bigoplus_{h \in G[X]} h$. In particular, $\mathcal{C}$ is nilpotent of nilpotency class 2 .

Lemma 5.1 (i) The action of the group $G$ by left (or right) tensor multiplication on the set $\operatorname{Irr}(\mathcal{C})-G$ is transitive.

(ii) There exists a normal subgroup $\Gamma$ of $G$ such that $G[X]=\Gamma$, for all non-invertible simple objects $X$ of $\mathcal{C}$. In particular $\operatorname{cd}(\mathcal{C})=\{1, \sqrt{|\Gamma|}\}$.

(iii) $\operatorname{Irr}(\mathcal{C})=G \cup\left\{X_{S} \mid s \in G / \Gamma\right\}$, where $X_{\bar{g}}=g \otimes X, g \in G$, obeying

$$
g \otimes h \simeq g h, \quad X_{\bar{g}} \otimes X_{\bar{h}}^{*} \simeq \bigoplus_{a \in \Gamma} g a h^{-1}, \quad \forall g, h \in G .
$$

Proof Let $X, Y \in \operatorname{Irr}(\mathcal{C})-G$. By assumption, $X \otimes Y^{*}=\bigoplus_{h \in S} h$ for some subset $S \subseteq G$. Then there exists $h \in G$ such that $m\left(h, X \otimes Y^{*}\right)>0$. Hence $m(X, h \otimes Y)=$ $m\left(h, X \otimes Y^{*}\right)=1$ and thus $h \otimes Y=X$. This shows that the left action of $G$ is transitive. The statement for the right action is proved similarly. This shows (i).

Part (ii) follows from transitivity of the right action, since $G[X \otimes h]=G[X]$, for all simple objects $X$, and for all $h \in G$. Note that, if $h \in G, \Gamma=G[h \otimes X]=h G[X] h^{-1}=$ $h \Gamma h^{-1}$. Hence $\Gamma$ is normal in $G$.

Finally, let $X$ be a fixed non-invertible simple object and set $X_{\bar{g}}=g \otimes X$, for every $\bar{g} \in G / \Gamma$. The isomorphism class of $X_{\bar{g}}$ is well defined, since $\Gamma=G[X]$. This also implies that $X_{\bar{g}} \simeq X_{\bar{h}}$ if and only if $\bar{g}=\bar{h}$ in $G / \Gamma$. It is clear that the relations (6) are satisfied. By (i), every non-invertible $Y \in \operatorname{Irr}(\mathcal{C})$ is isomorphic to $X_{\bar{g}}$, for some $g \in G$, and thus we get (iii).

Remark Let $G$ and $\Gamma$ be the groups associated to $\mathcal{C}$ as in Lemma 5.1. We shall say that $\mathcal{C}$ has generalized Tambara-Yamagami fusion rules of type $(G, \Gamma)$. In this case, the lemma implies that the rank of $\mathcal{C}$ is $[G: \Gamma](1+|\Gamma|)$ and $\operatorname{FPdim} \mathcal{C}=2|G|$.

In addition, if the index of $\Gamma$ in $G$ is odd, then $\mathcal{C}$ has a non-invertible simple object of order 2 . 
Tambara-Yamagami categories and Moore-Read categories are examples of fusion categories with generalized Tambara-Yamagami fusion rules of types $(G, G)$, where $G$ is a finite Abelian group, and $\left(\mathbb{Z}_{4}, \mathbb{Z}_{2}\right)$, respectively.

Proposition 5.2 Suppose $\mathcal{C}$ has generalized Tambara-Yamagami fusion rules of type $(G, \Gamma)$. Then we have:

(i) The adjoint subcategory $\mathcal{C}_{\text {ad }}$ coincides with $\mathcal{C}[\Gamma]$ and it is equivalent to the category of $\Gamma$-graded vector spaces.

(ii) The group $U(\mathcal{C})$ is of order $2[G: \Gamma]$.

(iii) The universal grading $\lambda: \operatorname{Irr}(\mathcal{C}) \rightarrow U(\mathcal{C})$ induces an isomorphism $G / \Gamma \simeq \lambda(G)$, such that $[U(\mathcal{C}): \lambda(G)]=2$.

(iv) Let $g \in U(\mathcal{C})$. Then the rank of $\mathcal{C}_{g}$ equals $|\Gamma|$ if and only if $g \in \lambda(G)$, and in this case $\mathcal{C}_{g}=(\tilde{g} \Gamma)$, where $\lambda(\widetilde{g})=g$. Otherwise, $\mathcal{C}_{g}$ is of rank 1 .

Proof (i) By Lemma 5.1 (ii), $X \otimes X^{*} \simeq \bigoplus_{h \in \Gamma} h$, for all non-invertible simple objects $X$ of $\mathcal{C}$. Therefore $\mathcal{C}_{\text {ad }}=\mathcal{C}[\Gamma]$. On the other hand, if $X$ is a non-invertible simple object, then $X$ gives rise (via left tensor multiplication) to a fiber functor on $\mathcal{C}[\Gamma]$. Then $\mathcal{C}[\Gamma]$ is equivalent to the category of $\Gamma$-graded vector spaces. This shows (i).

(ii) Since $\operatorname{FPdim} \mathcal{C}=|\Gamma||U(\mathcal{C})|=2|G|,|U(\mathcal{C})|=2[G: \Gamma]$, as claimed.

(iii) It is clear that $\lambda$ induces a group homomorphism $\lambda: G \rightarrow U(\mathcal{C})$. Since $\mathcal{C}_{\text {ad }}=\mathcal{C}[\Gamma]$, $\operatorname{ker} \lambda=\Gamma$ and $G / \Gamma \simeq \lambda(G)$. The last assertion follows from (ii).

(iv) We have FPdim $\mathcal{C}_{g}=|\Gamma|$, for all $g \in U(\mathcal{C})$. This implies (iv), in view of (iii) and Lemma 5.1 (ii).

Recall that a braided fusion category $\mathcal{T}$ is called Tannakian if there exists an equivalence of braided tensor categories $\mathcal{T} \simeq \operatorname{rep} G$, where $G$ is a finite group.

Lemma 5.3 Let $\mathcal{C}$ be a modular category with generalized Tambara-Yamagami fusion rules. Then we have:

(i) $\Gamma \simeq \mathbb{Z}_{2}$ and $\mathcal{C}[\Gamma]$ is not Tannakian.

(ii) $\mathcal{C}$ has a non-invertible simple object of order 2.

Proof (i) Since $\mathcal{C}$ is modular, $G \simeq U(\mathcal{C})$. From Proposition 5.2, we get that $|\Gamma|=2$. Hence FPdim $X=\sqrt{2}$, for all non-invertible simple object $X$ of $\mathcal{C}$. Suppose on the contrary that $\mathcal{C}[\Gamma]$ is Tannakian, that is, $\mathcal{C}[\Gamma] \simeq \operatorname{rep} \mathbb{Z}_{2}$ as braided tensor categories. We may regard $\mathcal{C}$ a fusion subcategory of its Drinfeld center $\mathcal{Z}(\mathcal{C})$. It follows from 
[12, Proposition 2.10; 9, Theorem 4.18(i)] that $\mathcal{C}$ is a $\mathbb{Z}_{2}$-equivariantization of a (not necessarily braided) fusion category $\mathcal{D}$. In other words, there exists a fusion category $\mathcal{D}$ and an action of $\rho: \mathbb{Z}_{2} \rightarrow \operatorname{Aut}_{\otimes} \mathcal{D}$ by tensor autoequivalences, such that $\mathcal{C} \simeq \mathcal{D}^{\mathbb{Z}_{2}}$ as fusion categories.

The forgetful functor $F: \mathcal{D}^{G} \rightarrow \mathcal{D}$ is a tensor functor and the image under $F$ of every object of $\mathcal{C}[\Gamma]$ is a trivial object of $\mathcal{D}$. Let $\delta$ be the nontrivial element of $\Gamma \simeq \mathbb{Z}_{2}$. If $X$ is a non-invertible simple object of $\mathcal{C}$, then we know from Lemma 5.1 that $X \otimes X^{*} \simeq \mathbb{1}+\delta$. Thus $F\left(X \otimes X^{*}\right) \simeq F(X) \otimes F(X)^{*}=\mathbb{1}+\mathbb{1}$, which is clearly impossible; indeed, this implies that $F(X)$ is not simple, so $F(X) \otimes F(X)^{*}$ should have at least four nonzero simple summands.

(ii) Let $\mathcal{C}_{\mathrm{pt}}^{\prime}$ denote the Müger centralizer of $\mathcal{C}_{\mathrm{pt}}=\mathcal{C}[G]$ in $\mathcal{C}$. By [9, Corollary 3.27], [14, Corollary 6.8], we have $\mathcal{C}[G]^{\prime}=\mathcal{C}_{\text {ad }}=\mathcal{C}[\Gamma]$. In particular, $\mathcal{C}[\Gamma] \subseteq \mathcal{C}[G]$ coincides with the Müger center of $\mathcal{C}[G]$. Since, by Part (i), $\mathcal{C}[\Gamma]$ is not Tannakian, $\mathcal{C}[G]$ is slightly degenerate, and it follows from [12, Proposition 2.6 (ii)] that $\mathcal{C}[G] \simeq \mathcal{C}[\Gamma] \otimes \mathcal{B}$ as braided tensor categories, where $\mathcal{B}$ is a pointed modular category. In particular, $|G|=2 \mathrm{FPdim} \mathcal{B}$. Let $\mathcal{B}^{\prime}$ be the Müger centralizer of $\mathcal{B}$ in $\mathcal{C}$. Since $\mathcal{C}$ is modular, we have $F P \operatorname{dim} \mathcal{B} F P \operatorname{dim} \mathcal{B}^{\prime}=\operatorname{FPdim} \mathcal{C}=2|G|$. Hence FPdim $\mathcal{B}^{\prime}=4$ and therefore $\mathcal{B}^{\prime}$ is an Ising category. Then $\mathcal{B}^{\prime}$, and thus also $\mathcal{C}$, has a non-invertible simple object of order 2 and (ii) holds. This finishes the proof of the lemma.

The following theorem is the main result of this section. Combined with the results in [9, Section 2.11 and Appendix B] and [24], it gives the classification of modular categories with generalized Tambara-Yamagami fusion rules.

Theorem 5.4 Let $\mathcal{C}$ be a modular category. Then $\mathcal{C}$ has generalized TambaraYamagami fusion rules if and only if $\mathcal{C} \simeq \mathcal{I} \otimes \mathcal{B}$, where $\mathcal{I}$ is an Ising category and $\mathcal{B}$ is a pointed modular category.

Proof Suppose that $\mathcal{C}=\mathcal{I} \otimes \mathcal{B}$, where $\mathcal{I}$ is an Ising category and $\mathcal{B}$ is a pointed category. Then every simple object of $\mathcal{C}$ is isomorphic to $Y \otimes g$, where $Y \in \operatorname{Irr}(\mathcal{I})$ and $g \in G(\mathcal{B})$. Then $\mathcal{C}$ is not pointed, and the non-invertible simple objects of $\mathcal{C}$ are represented by $X \otimes g$, where $X$ is the unique non-invertible simple object of $\mathcal{I}$ and $g \in G(\mathcal{B})$. This implies that $\mathcal{C}$ has generalized Tambara-Yamagami fusion rules of type $\left(G(\mathcal{B}), \mathbb{Z}_{2}\right)$. If in addition $\mathcal{B}$ is modular category, then so is $\mathcal{C}$. This proves the 'if' direction.

Conversely, suppose that $\mathcal{C}$ is modular and has generalized Tambara-Yamagami fusion rules. As in the proof of Lemma $5.3, \mathcal{C}[G] \simeq \mathcal{C}[\Gamma] \otimes \mathcal{B}$ as braided tensor categories, where $\mathcal{B}$ is a pointed modular category such that $\mathcal{I}:=\mathcal{B}^{\prime}$ is an Ising category. Moreover, 
$\mathcal{B}^{\prime}$ is also modular and $\mathcal{C} \simeq \mathcal{B} \otimes \mathcal{I}$ as modular tensor categories, by Müger [20, Theorem 4.2]. This finishes the proof of the theorem.

\section{Modularization of braided Tambara-Yamagami categories}

Throughout this section, $\mathcal{C}=\mathcal{T Y}(G, \tau, \chi)$ will be a Tambara-Yamagami fusion category, where $G$ is a finite Abelian group, $\tau$ is a square root of the order of $G$ in $k$ and $\chi: G \times G \rightarrow k^{\times}$is a non-degenerate symmetric bicharacter on $G$ [28].

We assume that $\mathcal{C}$ is braided. All possible structures of braided category in $\mathcal{C}$ are classified in [26]. In particular, $G$ is an elementary Abelian 2-group, and there are two choices of compatible ribbon structures. Let us consider a fixed choice $\theta \in \operatorname{Aut}\left(\mathrm{id}_{\mathcal{C}}\right)$, so that $\mathcal{C}$ becomes a premodular category.

Let $\mathcal{C}^{\prime} \subseteq \mathcal{C}$ be Müger center of $\mathcal{C}$. In the terminology of [5], $\mathcal{C}^{\prime}$ is the fusion subcategory of transparent objects of $\mathcal{C}$.

Lemma 6.1 Suppose $\mathcal{C}$ is not symmetric. Then we have $\mathcal{C}^{\prime}=\mathcal{C}[T]$, where $T$ is the subgroup of $G$ defined by $T=\{g \in G \mid \chi(g, g)=1\}$. Moreover, the category $\mathcal{C}$ is modularizable.

Proof Since $\mathcal{C}$ is not symmetric, and $X$ generates $\mathcal{C}, X \notin \mathcal{C}^{\prime}$. Hence $\mathcal{C}^{\prime} \subseteq \mathcal{C}[G]$.

Observe that an object $Z$ belongs to $\mathcal{C}^{\prime}$ if and only if $Z$ centralizes $X$. It follows from [26, Section 3.1] that, after a suitable normalization, the braidings $\sigma_{g, X}: g \otimes X \rightarrow X \otimes g$ and $\sigma_{X, g}: X \otimes g \rightarrow g \otimes X$ correspond, under the identification $g \otimes X=X=X \otimes g$, to $s(g) \mathrm{id}_{X}$, where $s(g) \in k^{\times}$are such that $s(g)^{2}=\chi(g, g)$, for all $g \in G$. This implies that $\mathcal{C}^{\prime}=\mathcal{C}[T]$, where $T=\{g \in G \mid \chi(g, g)=1\}$, as claimed.

Let $\theta \in \operatorname{Aut}\left(\mathrm{id}_{\mathcal{C}}\right)$ be the ribbon structure of $\mathcal{C}$. Then $\theta_{g}=s(g)^{2}$ [26, Section 3.5], for all $g \in G$. Hence $\theta_{g}=1$ for all $g \in T$ (this can also be deduced from [19, Lemma 5.4], since all simple objects $g$ of $\mathcal{C}^{\prime}$ are invertible and satisfy $g \otimes X \simeq X$ ). This implies that $\mathcal{C}^{\prime}$ is Tannakian and thus $\mathcal{C}$ is modularizable [5, Théorème 3.1].

Remark Suppose that $\mathcal{C}$ is symmetric. Since every invertible object $g$ of $\mathcal{C}$ satisfies $g \otimes X \simeq X$, then [19, Lemma 5.4] implies that $\mathcal{C}$ is Tannakian.

Let $\widehat{T}$ be the dual group of $T$, so that we have a non-canonical isomorphism of groups $T \simeq \widehat{T}$. In view of Lemma 6.1 , we have $\mathcal{C}[T] \simeq \operatorname{rep} \widehat{T}$, as symmetric tensor categories. Let $F: \mathcal{C} \rightarrow \widetilde{\mathcal{C}}$ denote the modularization functor, where $\widetilde{\mathcal{C}}$ is a modular category and $F$ is a dominant braided tensor functor. Observe that $\operatorname{FPdim} \tilde{\mathcal{C}}=\operatorname{FPdim} \mathcal{C} /|T|=2[G: T]$, and therefore $\operatorname{FPdim} \widetilde{\mathcal{C}}=2$ or 4 .

We have in addition: 
Proposition 6.2 There is an action $\rho: \underline{\widehat{T}} \rightarrow \underline{\text { Aut }} \otimes \overrightarrow{\mathcal{C}}$ by braided autoequivalences such that $\mathcal{C} \simeq \widetilde{\mathcal{C}}^{\hat{T}}$ as braided tensor categories over rep $\widehat{T}$.

Proof It follows from the results in [5] that there is an exact sequence of braided tensor functors

$$
\operatorname{rep} \widehat{T} \rightarrow \mathcal{C} \stackrel{F}{\rightarrow} \tilde{\mathcal{C}}
$$

Then the proposition follows from Bruguières and Natale [6, Corollary 5.31]. See [6, Example 5.33].

Remark Lemma 6.1 implies that if $\mathcal{C}$ is modular then $\chi(g, g) \neq 1$, for all $1 \neq g \in G$. On the other hand, if $\chi(g, g)=1$, for all $g \in G$, then either $\mathcal{C}$ is symmetric or $\tilde{\mathcal{C}}$ is pointed and $\operatorname{FPdim} \widetilde{\mathcal{C}}=2$.

Lemma 6.3 Suppose $\tilde{\mathcal{C}}$ is not pointed. Then $G(\widetilde{\mathcal{C}}) \simeq G / T \simeq \mathbb{Z}_{2}$.

Proof By Proposition 6.2, $\mathcal{C} \simeq \widetilde{\mathcal{C}}^{\hat{T}}$ is a $\widehat{T}$-equivariantization. The modularization functor corresponds to the forgetful functor $F: \widetilde{\mathcal{C}}^{\widehat{T}} \rightarrow \widetilde{\mathcal{C}}$. Since, by assumption, $\widetilde{\mathcal{C}}$ is not pointed, $T \neq G$.

Note that $\mathcal{C}^{\prime}=\mathcal{C}[T]$ is the kernel of $F$ in the sense of [6]. Then $F(G) \simeq G / T$ is isomorphic to a subgroup $G(\widetilde{\mathcal{C}})$.

Let $h \in G(\widetilde{\mathcal{C}})$ be an invertible object. We claim that $m(h, F(X))=0$. This can be seen as follows. Let $L: \mathcal{C} \rightarrow \mathcal{C}^{G}$ denote the left adjoint of $F$. Then we have $F L(h)=\oplus_{t \in \hat{T}} \rho^{t}(h)[6]$, and in particular, $F L(h)$ belongs to $\tilde{\mathcal{C}}_{\mathrm{pt}}$. Suppose on the contrary that $m(h, F(X))>0$. It follows by adjunction that $X$ is a simple direct summand of $L(h)$ and therefore $F(X)$ is a direct summand of $F L(h)$. This implies that $F(X) \in \widetilde{\mathcal{C}}_{\mathrm{pt}}$ and then, by surjectivity of $F, \widetilde{\mathcal{C}}_{\mathrm{pt}}=\widetilde{\mathcal{C}}$, since $X$ generates $\mathcal{C}$. This contradiction shows that $m(h, F(X))=0$, as claimed.

By surjectivity of the functor $F$, there exists $g \in G$ such that $m(h, F(g))>0$. Then $h \simeq F(g) \in G / T$. This shows that $G(\tilde{\mathcal{C}}) \simeq G / T$.

Observe next that, since $G$ is an elementary Abelian 2-group, $\chi$ induces a group homomorphism $f: G \rightarrow \mathbb{Z}_{2}$, defined in the form $f(g)=\chi(g, g)$, for all $g \in G$. We have $T=\operatorname{ker} f$, whence $[G: T]=2$, because $T \neq G$. 
Theorem 6.4 Let $\mathcal{C}$ be a braided Tambara-Yamagami fusion category and let $\tilde{\mathcal{C}}$ be the modularization of $\mathcal{C}$. Then we have:

(i) $\mathcal{C}$ is integral if and only if $\tilde{\mathcal{C}}$ is pointed. In this case $G(\tilde{\mathcal{C}})$ is of order 1,2 or 4.

(ii) Suppose that $\mathcal{C}$ is not integral. Then $\tilde{\mathcal{C}} \simeq \mathcal{I}$, as braided tensor categories, where $\mathcal{I}$ is an Ising category.

Note in addition that the integrality of $\mathcal{C}$ is determined by the parity of the rank of $G$, namely, $\mathcal{C}$ is integral if and only if the rank of $G$ is even.

Proof (i) We have an exact sequence of fusion categories $\mathcal{C}[T] \rightarrow \mathcal{C} \rightarrow \widetilde{\mathcal{C}}$. By [6] $\mathcal{C}$ is integral if and only if $\tilde{\mathcal{C}}$ is integral. If $\tilde{\mathcal{C}}$ is not pointed, then by Theorem 5.4, it contains an Ising subcategory and therefore it is not integral. Hence $\widetilde{\mathcal{C}}$ is integral if and only if it is pointed. Moreover, unless $\mathcal{C}$ is symmetric, in which case FPdim $\widetilde{\mathcal{C}}=1$, we have $\operatorname{FPdim} \tilde{\mathcal{C}}=2[G: T]=2$ or 4 . This shows (i).

(ii) Since $\mathcal{C}$ is not integral, $\widetilde{\mathcal{C}}$ is not integral either. In particular, $\tilde{\mathcal{C}}$ is not pointed. By Lemma 6.3 we have $G(\widetilde{\mathcal{C}}) \simeq G / T \simeq \mathbb{Z}_{2}$. The fusion rules of $\mathcal{C}$ imply that $\tilde{\mathcal{C}}$ has generalized Tambara-Yamagami fusion rules. Theorem 5.4 implies that $\widetilde{\mathcal{C}}$ is equivalent to an Ising category. This proves (ii) and finishes the proof of the theorem.

\section{References}

[1] H H Andersen, J Paradowski, Fusion categories arising from semisimple Lie algebras, Comm. Math. Phys. 169 (1995) 563-588 MR1328736

[2] B Bakalov, A Kirillov, Jr, Lectures on tensor categories and modular functors, University Lecture Series 21, American Mathematical Society (2001) MR1797619

[3] J Bichon, S Natale, Hopf algebra deformations of binary polyhedral groups, Transform. Groups 16 (2011) 339-374 MR2806496

[4] P Bonderson, Non-abelian anyons and interfermetry, $\mathrm{PhD}$ thesis, California Institute of Technology (2007)

[5] A Bruguières, Catégories prémodulaires, modularisations et invariants des variétés de dimension 3, Math. Ann. 316 (2000) 215-236 MR1741269

[6] A Bruguières, S Natale, Exact sequences of tensor categories, Int. Math. Res. Not. 2011 (2011) 5644-5705 MR2863377

[7] S Burciu, Kernels of representations and coideal subalgebras of Hopf algebras, Glasg. Math. J. 54 (2012) 107-119 MR2862388

[8] V Drinfeld, S Gelaki, D Nikshych, V Ostrik, Group-theoretical properties of nilpotent modular categories arXiv:0704.0195 
[9] V Drinfeld, S Gelaki, D Nikshych, V Ostrik, On braided fusion categories, I, Selecta Math. 16 (2010) 1-119 MR2609644

[10] P Etingof, D Nikshych, V Ostrik, On fusion categories, Ann. of Math. 162 (2005) 581-642 MR2183279

[11] P Etingof, D Nikshych, V Ostrik, Fusion categories and homotopy theory, Quantum Topol. 1 (2010) 209-273 MR2677836

[12] P Etingof, D Nikshych, V Ostrik, Weakly group-theoretical and solvable fusion categories, Adv. Math. 226 (2011) 176-205 MR2735754

[13] P Etingof, V Ostrik, Module categories over representations of $\mathrm{SL}_{q}(2)$ and graphs, Math. Res. Lett. 11 (2004) 103-114 MR2046203

[14] S Gelaki, D Nikshych, Nilpotent fusion categories, Adv. Math. 217 (2008) 1053-1071 MR2383894

[15] S-m Hong, E Rowell, On the classification of the Grothendieck rings of non-self-dual modular categories, J. Algebra 324 (2010) 1000-1015 MR2659210

[16] I M Isaacs, Character theory of finite groups, Pure and Applied Mathematics 69, Academic Press [Harcourt Brace Jovanovich Publishers], New York (1976) MR0460423

[17] Y Kashina, Y Sommerhäuser, Y Zhu, On higher Frobenius-Schur indicators, Mem. Amer. Math. Soc. 181 (2006) viii+65 MR2213320

[18] J Liptrap, Generalized Tambara-Yamagami categories, to appear in Journal of Algebra (2010) arXiv:1002.3166v2

[19] M Müger, Galois theory for braided tensor categories and the modular closure, Adv. Math. 150 (2000) 151-201 MR1749250

[20] M Müger, On the structure of modular categories, Proc. London Math. Soc. 87 (2003) 291-308 MR1990929

[21] S Natale, Hopf algebra extensions of group algebras and Tambara-Yamagami categories, Algebr. Represent. Theory 13 (2010) 673-691 MR2736029

[22] S-H Ng, P Schauenburg, Frobenius-Schur indicators and exponents of spherical categories, Adv. Math. 211 (2007) 34-71 MR2313527

[23] V Ostrik, Fusion categories of rank 2, Math. Res. Lett. 10 (2003) 177-183 MR1981895

[24] F Quinn, Group categories and their field theories, from: "Proceedings of the Kirbyfest”, (J Hass, M Scharlemann, editors), Geom. Topol. Monogr. 2 (1999) 407-453 MR1734419

[25] E Rowell, R Stong, Z Wang, On classification of modular tensor categories, Comm. Math. Phys. 292 (2009) 343-389 MR2544735

[26] J Siehler, Braided near-group categories arXiv:math/0011037 
[27] J Siehler, Near-group categories, Algebr. Geom. Topol. 3 (2003) 719-775 MR1997336

[28] D Tambara, S Yamagami, Tensor categories with fusion rules of self-duality for finite abelian groups, J. Algebra 209 (1998) 692-707 MR1659954

[29] J Thornton, On braided near-group categories arXiv:1102.4640v1

[30] V G Turaev, Quantum invariants of knots and 3-manifolds, de Gruyter Studies in Mathematics 18, Walter de Gruyter \& Co., Berlin (1994) MR1292673

Facultad de Matemática, Astronomía y Física, Universidad Nacional de Córdoba, CIEM-CONICET, Medina Allende s/n, Ciudad Universitaria 5000 Córdoba, Argentina

natale@famaf .unc.edu.ar

http://www.famaf.unc.edu.ar/ natale/

Received: 7 October $2011 \quad$ Revised: 29 October 2012 